\title{
The Errors Estimate of the Multistage Combined Investment Risk Assessment*
}

\author{
Yu Jike Zhou Zongfang ${ }^{1}$ \\ School of Management and Economics, University of Electronic Science \& Technology \\ Chengdu Sichuan, 610054, P.R.China
}

\begin{abstract}
Investment risk is economic development faced serious risk. The multistage combination investment risk assessment (MCIRA) can reduce the assessment error, but how to survey the error which produces by the MCIRA models, has the important significance. From theoretical side, the errors upper-bound of the MCIRA models is determined in this paper. We also give the relationships between the errors of the general MCIRA models, the simple average models and the errors of each investment risk assessment model in the combination.
\end{abstract}

Keywords: investment risk; errors estimate; simple average models; MCIRA models.

${ }^{*}$ This research has been supported by National Natural Science Foundation of China (No. 70971015).

'Zhou Zongfang, professor, zhouzf@uestc.edu.cn 


\section{Introduction}

Since the combined investment risk assessment methods were brought forward, a number of scholar have studied the manifold methods of determining the combined weighted coefficients, such as: the equal-weight or the simple average method, the recursive equal-weight method, the superiority-matrix method, novel particle swarm, real options and so on ${ }^{[1]-[8]}$. To our great regret, there were few research results available on the errors bounds of multistage combined investment risk assessment problems ${ }^{[9]-[10]}$.

In this paper, we first determine the errors maximum of the simple average combined investment risk assessment model, then the errors maximum of the general multistage combined investment risk assessment model will be discussed, at last we will give the relationships between the errors of the general multistage combined investment risk assessment (MCIRA) model and the simple average models and the errors of each investment risk assessment model in the combined assessment.

We utilize $n$ investment risk assessment models for the same investment risk assessment problem within $T$ given stages. To determining the combined risk assessment weights, the multistage combined investment risk assessment (MCIRA) problem is described as below:

On the assumption that $y_{t}(t=1,2, \ldots, T)$ are actual observations of investment risk in the $t$-th stage; fit $(t=1,2, \ldots, T ; i=1,2, \ldots, n)$ are the risk assessment values in advance of the $i$-th investment risk assessment model; $e_{i t}=y_{t}-f_{t}$ are errors between the observations and risk assessment values of the $i$-th model in the $t$-th stage. The combined investment risk assessment values are:

$$
f_{t}=\sum_{i=1}^{n} \mu_{i} f_{i t}, t=1,2, \ldots, T
$$

where $\mu_{i}(i=1,2, \ldots, n)$ are called the combined risk assessment weights, which satisfies $\sum_{i=1}^{n} \mu_{i}=1,(i=1,2, \ldots$, $n) ; e_{t}=\sum_{i=1}^{n} e_{i t}=y_{t}-f_{t}(t=1,2, \ldots, T)$ are called errors of the combined risk assessment.

$$
\begin{gathered}
\text { Let } \quad J=\sum_{t=1}^{T} e_{t}^{2}=\sum_{t=1}^{T}\left(\sum_{i=1}^{n} \mu_{i} e_{i t}\right)^{2} \\
=\sum_{i=1}^{n} \sum_{j=1}^{n}\left[\mu_{i} \mu_{j}\left(\sum_{t=1}^{T} e_{i t} e_{j t}\right)\right]=\mu^{\prime} E_{(n)} \mu
\end{gathered}
$$

be the errors square sum of the MCIRA model, where $\mu=\left(\mu_{1}, \mu_{2}, \cdots, \mu_{n}\right)^{\prime} \quad$ is called weight vector; $E_{i}=\left(e_{i 1}, e_{i 2}, \cdots, e_{i T}\right)^{\prime}(i=1,2, \ldots, n)$ are error vectors of the $i$-th model, $E_{i j}=E_{j i}=E_{i}^{\prime} E_{j}$, $E_{(n)}=\left(E_{i j}\right)_{n \times n}$ is a $n \times n$ symmetric matrix, $E_{i i}=\sum_{i=1}^{T} e_{i t}^{2}=J_{i}(i=1,2, \ldots, n)$ is just the investment risk assessment errors square sum of the $i$-th investment risk assessment model.

The matrix $E_{(n)}$ provides the error messages of each investment risk assessment model. We call $E_{(n)}$ the risk assessment errors information matrix of the MCIRA model and assume the matrix $E_{(n)}$ is invertible (or replace by the errors vectors $E_{i}(i=1,2, \ldots, n)$ are linear independence).

Definition 1: If $J^{*}=\left(\mu^{*}\right)^{\prime} E_{(n)} \mu^{*},\left(\mu_{i}^{*} \geq 0\right.$, $i=1,2, \ldots, n)$ is minimal about $\mu$, the $\mu^{*}$ is called the optimal combined assessment weight vector, the $J^{*}$ is called the minimal errors square sum of the optimal MCIRA model.

\section{The errors bounds of simple average model}

Definition 2: In equation (1), if $\mu_{i}=\frac{1}{n}, i=1,2, \ldots$, $n$, then the corresponding MCIRA model is called the simple average model.

Let errors square sum of simple average model is:

$$
J_{A}=\left(\mu^{(0)}\right)^{\prime} E_{(n)} \mu^{(0)}
$$

where $\mu^{(0)}=\left(\frac{1}{n}, \frac{1}{n}, \cdots, \frac{1}{n}\right)^{\prime}$.

If the errors square sum of the $i$-th investment risk assessment model is $J_{i}$, let their maximum and minimum 
be respectively:

$$
J_{\max }=\max _{1 \leq i \leq n}\left\{J_{i}\right\} \text { and } J_{\min }=\min _{1 \leq i \leq n}\left\{J_{i}\right\} .
$$

Theorem 1: If the MCIRA model is the simple average model, then

$$
J_{A} \leq J_{\max }
$$

Corollary 1: If the MCIRA model is the simple average model, then $J_{A}<J_{\min }$ if and only if

$$
\sum_{i=1}^{n} \sum_{j=1}^{n} E_{i j}<n^{2} J_{\min }
$$

Theorem 2: Supposing $E_{(n)}$ is an positive definite matrix, if the errors square sum of each investment risk assessment model in the combination is the same constant $\mathrm{C}\left(\right.$ i.e. $\left.J_{i}=\mathrm{C}, \mathrm{i}=1,2, \ldots, n\right)$, then

$$
J_{A}<\mathrm{C}
$$

Above theorem indicates that the simple average model can reduce the assessment errors, if $J_{i}=\mathrm{C}$ (constant), $i=1,2, \ldots, n$. For example, under the hypothesis of theorem 2, if $E_{i}=\left(e_{i 1}, e_{i 2}, \cdots, e_{i T}\right)^{\prime}$, $i=1,2, \ldots, n$ are $n(T \geq n)$ orthogonal vectors in $T$-dimension space (i.e. $E_{(n)}=\left(E_{i j}\right)_{n \times n}=\left(E_{i}^{\prime} E_{j}\right)_{n \times n}$ is a scalar matrix, and its diagonal elements are constant $C$ ), then the errors square sum of simple average model is $\frac{1}{n} C$.

\section{The errors estimate of the general MCIRA models}

Let the errors square sum of the general MCIRA model (In order to distinguish other peculiar circumstances, said that it is the general MCIRA model) be:

$$
J=J(\mu)=\mu^{\prime} E_{(n)} \mu
$$

where $\mu$ is a weight vector.

Lemma: ${ }^{[1]}$ If $\xi_{1}, \xi_{2}, \cdots, \xi_{n}$ are $n$ different ( $n$-dimension) weight vectors, and constants

$$
\begin{gathered}
\alpha_{1}, \alpha_{2}, \cdots, \alpha_{n} \text { satisfy } \sum_{i=1}^{n} \alpha_{i}=1, \alpha_{i}>0, \text { then } \\
J\left(\sum_{i=1}^{n} \alpha_{i} \xi_{i}\right)<\sum_{i=1}^{n} \alpha_{i} J\left(\xi_{i}\right)
\end{gathered}
$$

or $\left(\sum_{i=1}^{n} \alpha_{i} \xi_{i}\right)^{\prime} E_{(n)}\left(\sum_{i=1}^{n} \alpha_{i} \xi_{i}\right)<\sum_{i=1}^{n} \alpha_{i}\left(\xi_{i} E_{(n)} \xi_{i}\right)$

Theorem 3: If $\mu=\left(\mu_{1}, \mu_{2}, \cdots, \mu_{n}\right)^{\prime}$ is a weight vector, and $\sum_{i=1}^{n} \mu_{i}=1, \mu_{i}>0$, then

$$
J=J(\mu)=\mu^{\prime} E_{(n)} \mu<\sum_{i=1}^{n} \mu_{i} J_{i} .
$$

In other words, the errors square sums of the general MCIRA model don't exceed the weight sum of errors square sums of each investment risk assessment models in the combination.

Corollary 2: If $\mu$ is any nonnegative weight vector, then

$$
J=\mu^{\prime} E_{(n)} \mu<J_{\max }
$$

ii)

$$
J_{A}<\frac{1}{n} \sum_{i=1}^{n} J_{i} \leq J_{\max }
$$

Form the above results, we know that the errors square sum of the general MCIRA model doesn't exceed the maximum of errors square sum of each model in the combination. We can reduce the investment risk assessment errors by combining assessment model. The general MCIRA model's errors that showed by errors square sum are bounded above, and the superbound is $J_{\text {max }}$.

\section{An Example}

Considering a general MCIRA model: the reciprocal variance weight multistage combined investment risk assessment model, its weight vector is: 


$$
\mu=\left(\mu_{1}, \mu_{2}, \cdots, \mu_{n}\right)^{\prime}=\frac{1}{\sum_{i=1}^{n} J_{i}}\left(\frac{1}{J_{1}}, \frac{1}{J_{2}}, \cdots, \frac{1}{J_{n}}\right)^{\prime},
$$

From theorem 3, its errors square sum $J^{*}$ satisfies inequation as below:

$$
\begin{aligned}
J^{*}<\sum_{i=1}^{n} \mu_{i} J_{i} & =\frac{1}{\sum_{i=1}^{n} \frac{1}{J_{i}}}\left(\frac{1}{J_{1}} J_{1}+\frac{1}{J_{2}} J_{2}+\cdots+\frac{1}{J_{n}} J_{n}\right) \\
& =\frac{n}{\sum_{i=1}^{n} \frac{1}{J_{i}}}=M_{c}
\end{aligned}
$$

Where $M_{c}$ is called the harmonic mean of $J_{1}, J_{2}, \ldots, J_{n}$. Consequently the errors square sum $J^{*}$ of the reciprocal variance weight model is smaller then the harmonic mean $M_{c}$ of each investment risk assessment model in the combination. In particular, if $J_{i}>0, i=1,2, \ldots, n$, since the harmonic mean don't exceed the arithmetic average value, we express:

$$
J^{*}<M_{C} \leq \frac{1}{n} \sum_{i=1}^{n} J_{i} \leq J_{\max }
$$

the above inequation is just inequation (11).

\section{Conclusions}

In this paper, we have estimated the errors boundary of the simple average method and the general MCIRA models, and indicated that the errors is the bounded to the above theoretically. We have also applied the mathematics analysis techniques to determine the existent maximum of the errors square sum of the general MCIRA models. This article research to carries on the appraisal accurately to the investment risk, dodges the investment risk effectively, has the very important theory and the practical significance. The research conclusion is also suitable for the general combination risk assessment domain, such as combination credit risk assessment ${ }^{[12]}$, insurance risk assessment, disaster risk assessment and so on. We certainly believe that these studies will be of a great significant theoretical value and potential practical significance in the risk management domain.

\section{References}

1. J.M.Bates and C.W.J.Granger, Combination of Forecasts, Journal of forecasting, 20(4)(1969)451-468.

2. J.I.Munoz; J.Contreras; J.Caamano and P.F. Correia, Risk Assessment of Wind Power Generation Project Investments Based on Real Options, PowerTech, 2009 IEEE Bucharest, June 28-July 2(2009).

3. Jun Sun,Wei Fang etc, Solving the multi-stage portfolio optimization problem with a novel particle swarm optimization, Expert Systems with Applications: An International Journal, 6(23)( 2011) 6726- 6735.

4. G.Q.Liu, Z.F.Zhou and Y.Shi, A Multi-Dimensional Forward Selection Method for Firms' Credit Sale , Computers \& Mathematics with Applications, 54(2007)1228-1233.

5. Z.F.Zhou,T.Y. Mu and Y.Shi, The Mathematical Structure on Credit Evaluation, Fur East Journal of Applied Mathematics,1(20)(2005)113-119.

6. X.W. Tang, Z.F.Zhou and Y.Shi, Multi-Objective Constrained Nonlinear Optimization: An ODE Approach, International Journal of Information, 7(4)(2004)487-497.

7. Z.F.Zhou and X.W.Tang, The Research on Ordering Structure of Credit, Journal of Systems Science and Information, 3(2)(2004)531-534.

8. X.W. Tang, Z.F.Zhou and Y.Shi, The Research of Variable Weights in Combined Forecasting, Computers \& Mathematics with Applications, 45(2003)723-730.

9. Z.F.Zhou and X.W.Tang, The Error Estimates of Continuous Variable Weight Combined Forecasting, Journal of Systems Science and Information, 3(1)(2003)235-242.

10. X.W. Tang, Z.F.Zhou and Y.Shi, The Errors Bounds of Combined Forecasting, Journal of Mathematical and Computer Modelling, 36(2002) 997-1005.

11. X.W.Tang, Forecast theory with its applications, (University of Electronic Science \& Technology of China publisher,1992).

12. Z.F.Zhou,Y.Zhang and L. Chen, Emerging technology enterprise credit risk evolution mechanism and evaluation method,(Science press,Beijing,2010). 